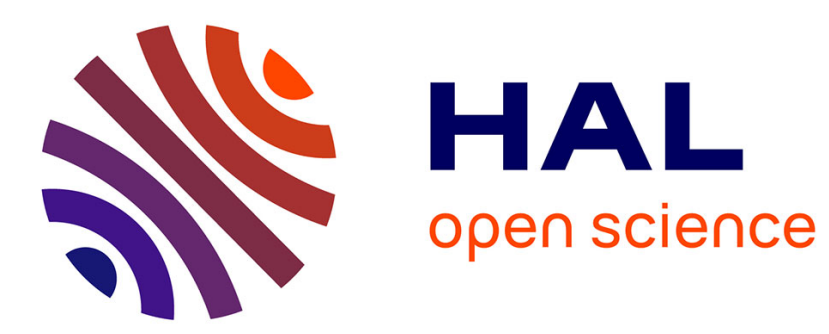

\title{
Nouvelles histoires de gendarmes et de policiers aux XIXe et XXe siècles. Regards sur l'historiographie récente des forces de l'ordre
}

\author{
Laurent Lopez, Jean-Noël Luc
}

\section{- To cite this version:}

Laurent Lopez, Jean-Noël Luc. Nouvelles histoires de gendarmes et de policiers aux XIXe et XXe siècles. Regards sur l'historiographie récente des forces de l'ordre. Histoire, économie et société, 2013, Histoire de la force publique aux XIXe et XXe siècles, 4, pp.3-19. hal-00927390

\section{HAL Id: hal-00927390 \\ https://hal.science/hal-00927390}

Submitted on 10 Apr 2017

HAL is a multi-disciplinary open access archive for the deposit and dissemination of scientific research documents, whether they are published or not. The documents may come from teaching and research institutions in France or abroad, or from public or private research centers.
L'archive ouverte pluridisciplinaire HAL, est destinée au dépôt et à la diffusion de documents scientifiques de niveau recherche, publiés ou non, émanant des établissements d'enseignement et de recherche français ou étrangers, des laboratoires publics ou privés. 


\section{NOUVELLES HISTOIRES DE GENDARMES ET DE POLICIERS AUX $\mathrm{XIX}^{\mathrm{E}}$ ET XX $^{\mathrm{E}}$ SIÈCLES REGARDS SUR L'HISTORIOGRAPHIE RÉCENTE DES FORCES DE L'ORDRE}

Laurent López, Jean-Noël Luc

Armand Colin | «Histoire, économie \& société »

2013/4 32e année | pages 3 à 19

ISSN 0752-5702

ISBN 9782200928377

Article disponible en ligne à l'adresse :

http://www.cairn.info/revue-histoire-economie-et-societe-2013-4-page-3.htm

\section{Pour citer cet article :}

Laurent López, Jean-Noël Luc, « Nouvelles histoires de gendarmes et de policiers aux $\mathrm{XIX}^{\mathrm{e}}$ et $\mathrm{XX}^{\mathrm{e}}$ siècles Regards sur l'historiographie récente des forces de l'ordre », Histoire, économie \& société 2013/4 (32e année), p. 3-19.

DOI 10.3917/hes.134.0003

Distribution électronique Cairn.info pour Armand Colin.

(C) Armand Colin. Tous droits réservés pour tous pays.

La reproduction ou représentation de cet article, notamment par photocopie, n'est autorisée que dans les limites des conditions générales d'utilisation du site ou, le cas échéant, des conditions générales de la licence souscrite par votre établissement. Toute autre reproduction ou représentation, en tout ou partie, sous quelque forme et de quelque manière que ce soit, est interdite sauf accord préalable et écrit de l'éditeur, en dehors des cas prévus par la législation en vigueur en France. Il est précisé que son stockage dans une base de données est également interdit. 


\title{
Nouvelles histoires de gendarmes et de policiers aux $\mathrm{XIX}^{\mathrm{e}}$ et $\mathrm{XX}^{\mathrm{e}}$ siècles Regards sur l'historiographie récente des forces de l'ordre
}

\author{
Laurent López et Jean-Noël Luc
}

\begin{abstract}
Résumé
L'abondance des titres disponibles a empêché d'étendre ce coup d'œil sur l'actualité de l'histoire de l'exercice de la police - policing en anglais - aux précieux apports de l'historiographie étrangère et des autres sciences humaines et sociales. Ce panorama montre néanmoins la fécondité de quelques grands chantiers, consacrés à la circulation internationale des savoirs, des dispositifs et des usages policiers, à l'émergence des systèmes policiers (en cours d'analyse dans le cadre du programme ANR SYSPOE), aux polices coloniales, un terrain de recherche particulièrement dynamique, à l'histoire sociale des hommes et des femmes qui donnent corps aux institutions policières, aux gendarmes, désormais pris en compte, ainsi qu'aux interrelations entre ces militaires originaux, chargés de fonctions policières, et les personnels des polices civiles, interrelations dont l'étude permet de commencer à construire une véritable histoire de la force publique.
\end{abstract}

\section{Abstract}

The abundance of available titles prevented to extend this look at the news in the history of policing to the valuable contributions of foreign historians and other academics in humanities and socialstudies. However, this view shows the fertility of some major projects, dedicated to the international circulation of knowledge, devices and police practices, the emergence of police systems (currently being analyzed as part of the ANR program SYSPOE), to colonial police forces, a particularly dynamic field of research, to the social history of men and women who give body to police forces, to the gendarmes, now taken into account, and to the interrelationships between these odd military, with policing duties, and the civilian police personnel, the study of all of which helps build a real story of policing. 
«Les faits du domaine de la police ont eu et auront, de tout temps, le privilège d'exciter la curiosité du public ${ }^{1} \gg .$. Mieux vaut tard que jamais : depuis deux décennies, la prémonition de cet officier de paix des années 1880 se vérifie, pour le monde académique, à propos des $\mathrm{XIX}^{\mathrm{e}}, \mathrm{XX}^{\mathrm{e}}$ et $\mathrm{XXI}^{\mathrm{e}}$ siècles. Il faut même être attentif pour ne rien rater de l'actualité éditoriale et scientifique autour d'un objet étudié dans des cadres disciplinaires, thématiques, chronologiques et géographiques de plus en plus diversifiés. Et ce foisonnement s'accompagne, depuis les années 2000, d'une extension des regards au-delà des forces civiles, les seules auxquelles les chercheurs s'étaient d'abord intéressés. Mais la simple arrivée des gendarmes dans le chantier ne signifie pas qu'il peut maintenant s'arrêter. Cinq ouvrages récents montrent, au contraire, qu'il progresse en franchissant avec bonheur une nouvelle étape : Police et gendarmerie dans l'Empire napoléonien (sous la direction de Jacques-Olivier Boudon); Servir Napoléon. Policiers et gendarmes dans les départements annexés, 1796-1814 (Aurélien Lignereux) : In Thrall to Political Change. Police and Gendarmerie in France (Malcolm Anderson); La Guerre des polices n'a pas eu lieu : gendarmes et policiers, coacteurs de la sécurité publique sous la Troisième République, 1870-1914 (Laurent López); Les Gendarmes belges, français et néerlandais à la sortie de la Seconde Guerre mondiale (Jonas Campion) ${ }^{2}$. L'innovation consiste ici à sortir d'un cadre national et/ou à cesser d'étudier séparément ou parallèlement les deux grands acteurs de la force publique. Jonas Campion croise réellement le destin des gendarmes de trois pays différents et les modes de rétablissement d'une légalité policière au cours d'une période critique, replacée, en plus, dans les évolutions des années 1920-1950. Les autres travaux rassemblent sur une grande échelle policiers et gendarmes dans une même histoire de la force publique. Grâce aux moyens humains et matériels dont il dispose, le chantier ANR Histoire des systèmes policiers en Europe, XVIII ${ }^{e}-X I X^{e}$ siècles (SYSPOE), ouvert le $1^{\text {er }}$ janvier 2013, et coordonné par Vincent Denis, peut aller plus loin en élargissant l'enquête à d'autres agents de la sécurité intérieure et en réunissant deux siècles habituellement séparés par les frontières académiques françaises.

La nouvelle histoire de l'exercice de la police s'intéresse légitimement à l'ensemble des acteurs (militaires ou civils, publics ou privés, professionnels ou non, institutionnalisés ou temporaires) chargés de cette mission, ainsi qu'à la participation de la Justice, de l'institution pénitentiaire, des services de renseignement, des corps de pompiers et des Douanes au maintien de l'ordre. Le poids des institutions policières nationales ne doit pas faire oublier les agents plus modestes (gardes champêtres ${ }^{3}$, gardes forestiers et, en terres coloniales, milices ou auxiliaires indigènes), surtout lorsqu'ils interviennent au cœur de la population longtemps la plus nombreuse, celle des campagnes. L'ouverture du champ d'étude concerne aussi la chronologie. D'une part, car la Révolution française n'a pas tout inventé : les fameuses brigades de gendarmerie, chères à l'ego-histoire du corps, sont instituées... en 1720. D'autre part, car le passage d'une police responsable de «tout ce qui comprend la sûreté et les commodités de la vie » de chaque cité, selon la définition de Portalis, à un instrument de l'État chargé du maintien de l'ordre et de la sécurité se produit tout au long des XVIII ${ }^{\mathrm{e}}$ et XIX ${ }^{\mathrm{e}}$ siècles. Et les polices de l'Ancien Régime méritent d'autant plus l'attention que les modernistes, engagés les premiers dans l'histoire de la criminalité

1. Anonyme, Vingt ans de police. Souvenirs et anecdotes d'un ancien officier de paix, Paris, E. Dentu, 1881 , p. 1.

2. Respectivement : Paris, éd. SPM, 2013 ; Seyssel, Champ Vallon, 2012 ; New York, Oxford University Press, 2011 ; Paris, PUPS, 2014 ; Bruxelles, André Versailles, 2011.

3. Fabien Gaveau, L'Ordre aux champs. Histoire des gardes champêtres en France, de la Révolution française à la Troisième République. Pour une autre histoire de l'État, doctorat, Dijon, dir. Jean-Marc Berlière, 2006. 
et de la Justice, ont commencé à étudier les acteurs de l'ordre, y compris la maréchaussée ${ }^{4}$, avant que des dix-neuvièmistes, puis des vingtièmistes, ne s'y intéressent.

La taille de ce panorama a cependant conduit à le resserrer autour des seuls XIX ${ }^{\mathrm{e}}$ et $\mathrm{XX}^{\mathrm{e}}$ siècles et des deux grandes forces publiques françaises, dont les évolutions spécifiques sur plusieurs registres - territorialisation, tutelle de l'État, bureaucratisation, spécialisation, militarisation ou civilianisation - ne permettent pas de réemployer uniquement les analyses forgées avec succès pour les époques antérieures. L'abondance des titres disponibles sur ce champ particulier ${ }^{5}$ interdit hélas toute prétention à l'exhaustivité. Et la même profusion empêche d'étendre l'enquête aux autres sciences humaines et sociales, ainsi qu'à la luxuriante production étrangère ${ }^{6}$, où les historiennes et les historiens français sont pourtant heureux de trouver des analyses fructueuses pour l'étude du passé ${ }^{7}$, de précieuses occasions de comparaison et des regards salutairement décentrés.

\section{Retour sur l'essor de l'histoire contemporaine de la Police}

Après l'initiative, restée isolée, du colloque de décembre 1983, Maintien de l'ordre et polices en France et en Europe au XIX ${ }^{e}$ siècle ${ }^{8}$, il faut attendre les publications de JeanMarc Berlière sur les polices de la Troisième République, et principalement sur les forces de la préfecture de police de Paris, pour trouver, à partir du début des années 1990, une série de jalons pionniers ${ }^{9}$. En complément de ces travaux, des monographies régionales permettent de découvrir les dispositifs de la force publique en province avec, en particulier, les thèses de Jean-François Tanguy sur l'Ille-et-Vilaine (1986), Marie-Thérèse Vogel sur les polices urbaines de Grenoble (1993) ou Édouard Ebel sur l'Alsace (1999) ${ }^{10}$. Ce décentrement

4. Jacques Lorgnier, Maréchaussée. Histoire d'une révolution administrative et judiciaire, Paris, L'Harmattan, 1994, 2 t., et Pascal Brouillet, La Maréchaussée dans la généralité de Paris (1718-1791). Étude institutionnelle et sociale, doctorat, EPHE, dir. Jean Chagniot, 2002.

5. Plusieurs instruments bibliographiques viennent heureusement au secours des chercheurs : Ilsen About, Bibliographie sur l'histoire de la police en Europe, 1600-1914 (sans classement), CIRSAP, http://irhis.recherche. univ-lille3.fr/ANR-CIRSAP.html ; Jean-Claude Farcy, « Présentation de la Bibliographie d'Histoire de la justice française (1789-2011) », Criminocorpus [en ligne], http://criminocorpus.cnrs.fr/outils/16581/; Xavier Rousseaux, « Historiographie du crime et de la justice criminelle dans l'espace français (1990-2005). Partie I : du Moyen-Âge à la fin de l'Ancien Régime », Crime, Histoire \& Sociétés, 2006/1, p. 123-158, http://chs.revues.org/index203. html ; «Partie II : de la Révolution au XXI ${ }^{\mathrm{e}}$ siècle », id., 2006/2, p. 123-161, http://chs.revues.org/index224.html ; Édouard Ebel, Ronan L'Héreec et Jean-Noël Luc, Bibliographie de l'histoire de la gendarmerie, Vincennes, SHD, 2011, http://www.servicehistorique.sga.defense.gouv.fr/Bibliographie-de-l-histoire-de-la.html

6. Produite, par exemple, dans le cadre des chantiers suivants : International Centre for the History of Crime, Policing and Justice (Open University), le vivier de la somme dirigée par Clive Emsley, The History of Policing, 4 vol., Abingdon, Ashgate, 2011, Centre d'histoire du droit et de la justice (Université catholique de Louvain), Villa Ten Hompel (Münster), équipe DAMOCLES (Université de Genève), équipe de Livio Antonielli (Milan et Messine), Groupe Européen de Recherche sur les Normativités (GERN).

7. Par exemple, dans les travaux du fondateur de la sociologie de la gendarmerie (François Dieu, Gendarmerie et modernité, étude de la spécificité gendarmique aujourd'hui, Paris, Montchrestien, 1993), comme dans ceux des pionniers de la sociologie de la police (par exemple, Jean-Louis Loubet del Bayle) et des autres sociologues de cette institution cités dans ce panorama.

9. Jean-Marc Berlière, L'Institution policière en France sous la III République (1870-1914), doctorat, Université de Bourgogne, dir. P. Lévêque, 1991 ; id., Le monde des polices en France, Bruxelles, Éd. Complexe, 1996.

10. Jean-François Tanguy, Le Maintien de l'ordre public en Ille-et-Vilaine, 1870-1914, doctorat, Rennes II, dir. J. Léonard, 1986 ; Marie-Thérèse Vogel, Les Polices des villes entre local et national, l'administration des polices urbaines sous la Troisième République, doctorat, Grenoble II, dir. François d'Arcy, 1993 ; Édouard Ebel, Police et société : histoire de la police et de son activité en Alsace au XIX siècle, Strasbourg, PUS, 1999. 
était nécessaire, puisque la police à Paris est, à la fois, représentative des structures ou des évolutions nationales et atypique.

Trois ouvrages, tous parus en 2012, ramènent l'attention sur la gestion policière des capitales, en l'occurrence Paris et Istanbul. Quentin Deluermoz retrace l'évolution de la figure de l'agent de police parisien au cours du second XIX ${ }^{\mathrm{e}}$ siècle. Son approche privilégie la manière dont l'espace urbain est pacifiquement investi par le représentant d'un ordre politique qui police la rue par ses contacts interpersonnels avec la population. Certaines des mutations mises au crédit de la Troisième République en matière de maintien de l'ordre commencent, en réalité, à la fin d'un Second Empire dont l'autoritarisme s'atténue ${ }^{11}$.

Christian Chevandier propose la première histoire, politique, civique et sociale, des gardiens de la paix, des années 1870 à nos jours, en ajoutant au noyau dur parisien quelques détours dans les communes du Havre et d'Oullins (Rhône). Avec un souci de la nuance, cet auteur appréhende le métier de ces policiers urbains à travers leur mission principale (discipliner des citadins réticents), l'évolution de leurs caractéristiques (profils, carrières, pratiques, identité, sociabilité, corporatisme) et leurs comportements diversifiés au cours de moments exceptionnels (les guerres, la crise des années 1930, l'Occupation, la Guerre d'Algérie ou le « printemps agité » de 1968) ${ }^{12}$.

Noémi Lévy-Aksu analyse la reconfiguration de la police d'Istanbul à l'époque d'Abdülhamid II puis des Jeunes-turcs, tous très préoccupés par le contrôle des populations dans un contexte d'explosion migratoire et de mouvements sociaux. Après avoir précisé l'intérêt porté aux solutions étrangères, et principalement françaises, elle montre que les transferts ne peuvent pas être compris sans prendre en considération les interprétations et le rôle des récepteurs. Son ouvrage s'achève avec bonheur sur l'étude des interactions entre les « agents intermédiaires » (veilleurs de nuit et caïds) et les habitants, qui éclairent, au-delà l'intervention des forces institutionnalisées, le « contrôle par le bas » exercé au niveau des quartiers $^{13}$.

Deux autres ouvrages récents, l'un rédigé et l'autre dirigé par Jean-Marc Berlière et René Lévy, témoignent également de la vitalité du chantier ${ }^{14}$. Un copieux volume de synthèse présente l'Histoire des polices en France, mais en ne développant pas celle de la gendarmerie, sauf à propos des réformes des vingt dernières années. Une réflexion collective est, par ailleurs, conduite sur les témoignages obtenus « quand des policiers se mettent à table », ici à l'occasion de quatre-vingt-quatorze entretiens, réalisés, à partir de 1991, par l'ancien Institut des hautes études de sécurité intérieure (IHESI) et par le CESDIP. On comprend mieux comment les apports respectifs des historiens et des sociologues améliorent la collecte et l'exploitation de cette source orale particulière. Malgré les limites de la mémoire, les informations recueillies sont indispensables pour éclairer des sujets délicats comme l'Occupation ou les guerres coloniales.

11. Quentin Deluermoz, Policiers dans la ville. La construction d'un ordre public à Paris (1854-1914), Paris, Publications de la Sorbonne, 2012.

12. Christian Chevandier, Policiers dans la ville. Une histoire des gardiens de la paix, Paris, Gallimard, 2012. Pour une comparaison avec l'Angleterre, Clive Emsley, The Great British Bobby : A history of British policing from 1829 to the present, London, Quercus, 2009.

13. Noémi Lévy-Aksu, Ordre et désordre dans l'Istanbul ottomane (1879-1909), Paris, Karthala, 2012.

14. Jean-Marc Berlière, René Lévy, Histoire des polices en France, de l'Ancien Régime à nos jours, Paris, Nouveau Monde Éditions, 2011 ; id. (dir.), Le Témoin, le sociologue et l'historien : quand des policiers se mettent à table, Paris, Nouveau Monde Éditions, 2010. 
Les productions récentes ou à venir bénéficient de « l'ouverture d'un moment historiographique » entre 2003 et 2007, repéré par Vincent Milliot à travers quatre publications ${ }^{15}$, et dont on peut se demander s'il s'est vraiment arrêté ! À côté de la parution de l'ouvrage de Paolo Napoli (2003), qui invite les chercheurs à étudier la police, non pas à partir des conceptualisations, mais à travers des pratiques situées dans l'espace et le temps ${ }^{16}$, ce moment s'est construit autour de plusieurs réalisations collectives. En 2003, puis en 2007, La Revue d'histoire moderne et contemporaine accorde un traitement privilégié (un numéro entier, puis un demi-numéro) aux thèmes respectifs des " Espaces policiers, $\mathrm{XVII}^{\mathrm{e}}-\mathrm{XX}^{\mathrm{e}}$ siècles », puis de «L'Ordre public ${ }^{17}$ ». En 2005, l'Histoire et dictionnaire de la police, $d u$ Moyen Âge à nos jours vulgarise, parfois, certains résultats des recherches récentes ${ }^{18}$, tandis qu'un groupe de chercheurs organise, à Caen, un colloque sur les ambitions réformatrices et la circulation des mémoires policiers du XVIII ${ }^{\mathrm{e}}$ siècle et du premier XIX ${ }^{\mathrm{e}}$ siècle $^{19}$. La même année, deux autres réalisations attirent l'attention sur le " défi gendarmique », selon la formule de Vincent Milliot : le colloque Soldats de la loi. La gendarmerie au XX siècle et la sortie d'un guide de recherche de plus de mille pages sur l'histoire de la maréchaussée et de la gendarmerie ${ }^{20}$. De plus, le chantier ANR Circulation et constructions des savoirs policiers européens, 1650-1850 (CIRSAP) commence ses travaux en 2006, tandis que le bouillonnement existe aussi à l'étranger, comme le montre, par exemple, la parution au cours de la même période de trois ouvrages collectifs sur les polices européennes au $\mathrm{XX}^{\mathrm{e}}$ siècle : Policing Interwar Europe. Continuity, Change and Crisis, 1918-40 (Gerald Blaney éd.), Conflict \& Legality. Policing mid-twentieth century Europe (Gerard Oram ed.) et The Impact of World War II on Policing in North-West Europe (Cyrille Fijnaut éd.) ${ }^{21}$. En 2007, terminus ad quem du panorama, deux initiatives contribuent à consolider le nouveau chantier. Jean-Marc Berlière lance un observatoire scientifique toujours opérationnel : la «Lettre aux amis de la police (et de la gendarmerie) ${ }^{22}$ ». Le colloque international $\hat{E} t r e$ policier : les métiers de police(s) en Europe, $X V I I I^{e}-X X^{e}$ siècles constitue une nouvelle étape pour l'histoire policière européenne en raison de son ampleur (39 communications sur 13 pays), de sa perspective résolument comparatiste et de son ouverture, chronologique, disciplinaire et institutionnelle (6 des communications traitent des gendarmeries) ${ }^{23}$.

15. Vincent Milliot, « Histoire des polices : l'ouverture d'un moment historiographique », Revue d'histoire moderne et contemporaine, $\mathrm{n}^{\circ}$ 54-2, 2007, p. 162-177.

16. Paolo Napoli, Naissance de la police moderne. Pouvoir, normes, sociétés, Paris, La Découverte, 2003.

17. Catherine Denys et Vincent Milliot (dir.), «Espaces policiers, $\mathrm{XVII}^{\mathrm{e}}-\mathrm{XX}^{\mathrm{e}}$ siècles », dans Revue d'histoire moderne et contemporaine, 50-1, 2003.

18. Histoire et dictionnaire de la police, du Moyen Âge à nos jours, dir. Michel Aubouin, Arnaud Teyssier, Jean Tulard, Paris, Robert Laffont, 2005.

19. Vincent Milliot (dir.), Les Mémoires policiers, 1750-1850. Écritures et pratiques policières du Siècle des Lumières au Second Empire, Rennes, PUR, 2006.

20. Jean-Noël Luc (dir.), Histoire de la maréchaussée et de la gendarmerie. Guide de recherches, MaisonsAlfort, SHGN, 2005, avec, en plus, un dictionnaire de 315 notices (en ligne sur le site du SHD) ; id., Soldats de la loi. La gendarmerie au $X X^{e}$ siècle, Paris, PUPS, 2010.

21. Londres, Francis Boutle Publishers, 2003 ; Louvain, Leuven University Press, 2004 ; New York, Plagrave Macmillan, 2007.

22. http://politeia.over-blog.fr/pages/Lettre_aux_amis_de_la_police_et_de_la_gendarmerie_2009052351051.html.

23. Jean-Marc Berlière, Catherine Denys, Dominique Kalifa, Vincent Milliot (dir.), Métiers de police. Être policiers en Europe, XVIII $-X X^{e}$ siècles, Rennes, PUR, 2008. 


\section{Les gendarmes appréhendés par Clio ou la longue histoire d'une force militaire originale}

L'héritière de la maréchaussée a été longtemps négligée par les historiens, à l'exception de quelques rares chercheurs anglo-saxons : Terry W. Strieter, Michael Broers ou Clive Emsley $^{24}$. Avant le début des années 2000, aucune université française ne lui avait consacré une manifestation scientifique, et aucune des grandes revues historiques, un article autonome. Le développement heureux de l'histoire des polices restait ici sans effets. D'une part, car l'étude des multiples forces civiles constituait déjà un vaste chantier attractif. D'autre part, car la confusion, dans le monde académique comme dans de nombreuses fictions, de la mission (la police) et de la principale institution civile et urbaine de l'époque contemporaine (la Police) maintenait les « soldats de la loi » à l'écart de certaines réalisations (colloques, expositions, publications). Trop militaire pour les historiens du social, trop civil pour les historiens de l'armée : le gendarme, relégué au mieux dans une note de bas de page, restait un objet historique non identifié.

Cette situation a changé grâce aux initiatives du Service historique de la gendarmerie nationale, SHGN (créé en 1995, puis absorbé à partir de 2005, malgré son dynamisme, par le Service historique de la Défense, SHD) et de Jean-Noël Luc, professeur à l'université Paris-Sorbonne. En 2000, cette université organise le premier colloque historique sur la gendarmerie (au XIX ${ }^{\mathrm{e}}$ siècle $)^{25}$, avant de signer, en 2001, une convention avec la Direction générale de la gendarmerie nationale (DGGN) pour développer la recherche sur l'histoire de cette institution. Entre 2000 et octobre 2013, 151 maîtrises, DEA ou mastères ainsi que 14 thèses ont été soutenus au sein d'un séminaire désormais intitulé Gendarmeries, polices et société : régulation sociale, ordre public, sécurité intérieure et Défense, XIX ${ }^{e}-X X I^{e}$ siècles, et codirigé avec Arnaud Houte. Au cours de la même période, ce chantier, intégré au Centre d'histoire du XIX ${ }^{\mathrm{e}}$ siècle (Paris I-Paris IV), a fourni la matière de 28 ouvrages sur l'histoire de la gendarmerie et (co) organisé 9 colloques et journées d'étude. Cette productivité est aussi le fruit d'un partenariat avec le SHGN, puis avec la Société nationale de l'histoire et du patrimoine de la gendarmerie (SNHPG), fondée par le général (2S) Georges Philippot, ancien chef du SHGN, et dont la revue, Force Publique, publie certains résultats des recherches universitaires.

L'histoire de la gendarmerie a aussi progressé grâce aux travaux réalisés dans d'autres universités (Bordeaux IV, Rennes II, Caen, Montpellier III, Dijon, Louvain, etc) et dans des IEP, comme ceux de Toulouse et d'Aix, au CESDIP, au SHGN et au SHD, pour ne citer que ces exemples. Il faut s'en réjouir. Si l'Université Paris-Sorbonne, traditionnellement engagée dans l'histoire de la défense et de la sécurité, était bien placée pour ouvrir le nouveau chantier, sa convention avec la DGGN ne s'accompagne d'aucune exclusivité. La gendarmerie, les armées, la police, appartiennent aux citoyens : leur histoire ne doit pas être pilotée par un établissement universitaire particulier.

Pourquoi s'intéresser à des militaires atypiques, statutairement non-combattants mais chargés d'assurer «le maintien de l'ordre et l'exécution des lois», selon la formule emblématique des textes organiques de la gendarmerie? Parce que leur histoire permet

24. Par exemple : Terry W. Strieter, « Drinking on the Job : Ivresse among the French Gendarmerie in the $\mathrm{XIX}^{\text {th }}$ Century », Proceedings of the Annual Meeting of the Western Society for French History, ${ }^{\circ} 13,1986$, p. 173-181 ; Michael Broers, « The Police and the Padron : Italian Notabili, French Gendarmes and the Origins of the Centralized State in Napoleonic Italy », European History Quaterly, n² 26, 1996, p. 331-353 ; Clive Emsley, Gendarmes and the State in Nineteenth-Century Europe, Oxford, OUP, 1999, 288 p. Sur l'oubli des gendarmes, voir Jean-Noël Luc, Gendarmerie, État et Société au XIX siècle, Paris, Presses de la Sorbonne, 2002, p. 10-21.

25. Ibid. 
d'étudier le fonctionnement de l'État sous un angle nouveau et de mieux comprendre comment s'inventent une police de proximité, un art de l'enquête, une technique du maintien de l'ordre ${ }^{26}$ et des participations spécifiques aux opérations militaires, à la colonisation et aux dispositifs de défense. En reconstituant le destin d'une communauté de 7500 personnes en 1791, 27000 en 1914 et plus de 100000 au début du XXI siècle (dont $15 \%$ de femmes), on peut analyser les voies de sa professionnalisation, suivre les aléas de ses relations avec la population ${ }^{27}$, y compris dans les villes, où elle est présente dès l'origine ${ }^{28}$, et avec les autres forces de l'ordre, expliquer l'articulation progressive de la militarité et de la culture du service public dans une partie du système policier français et, même, élargir le champ de l'histoire des femmes et du genre ${ }^{29}$. On comprend mieux, aussi, la place du bicorne puis du képi du gendarme dans l'imaginaire national ${ }^{30}$, de Pandore Une Femme d'honneur en passant par Nuit et Brouillard, les films de Pagnol, La tactique du gendarme (Bourvil), Hécatombe (Brassens), le Déserteur (Boris Vian) et Les Gendarmes de Saint-Tropez. L'objet d'étude est irremplaçable : jusqu'à l'étatisation des polices municipales, en 1941, la gendarmerie représente la seule police française «nationale », si l'on considère que ce terme désigne une force de l'État implantée sur l'ensemble du territoire. Publié en 2010, le doctorat d'Arnaud Houte, Le métier de gendarme au XIX siècle, ajoute à l'exploration des thèmes rappelés ici, une autre lecture, «à hauteur du bicorne », des transformations de l'État, de la vie publique et de la société ${ }^{31}$.

L'histoire de la gendarmerie, «partie intégrante de l'armée », selon la formule de l'ordonnance de 1820, enrichit aussi les travaux conduits sur les relations entre les institutions policières et militaires. Plusieurs pistes émergent sur ce nouveau front. L'analyse des processus de policiarisation ou de militarisation, depuis le niveau du maintien de l'ordre, le plus étudié, jusqu'au choix des uniformes, des armes et de la symbolique, négligés par la recherche académique, repère les influences réciproques, les panachages ou les oppositions entre « culture militaire et systèmes policiers ${ }^{32}$. » L'étude des polices en situation d'occupation, renouvelée notamment par les travaux de Jean-Marc Berlière, Benoît Marjerus et Jonas Campion, révèle leurs rapports particuliers avec les armées, le rôle catalyseur de ces périodes, génératrices d'innovations préparées en amont et parfois maintenues en aval, la poursuite des missions quotidiennes, en deçà des compromissions spectaculaires, enfin les enjeux et l'ampleur des épurations ${ }^{33}$. L'histoire des missions militaires de la gendarmerie,

26. Fabien Cardoni, La Garde républicaine d'une République à l'autre (1848-1871), Rennes, PUR, 2008, et Thierry Forest, La Gendarmerie mobile à l'épreuve de mai 1968, Vincennes, SHD, 2007.

27. Aurélien Lignereux, La France rébellionnaire, Les résistances à la gendarmerie (1800-1859), Rennes, PUR, 2008.

28. La Gendarmerie, force urbaine, du XVIII siècle à nos jours, actes publiés dans Force publique, $\mathrm{n}^{\circ} 3$, 2008, http://www.force-publique.net.

29. Anne Geesen, Une Femme d'honneur au miroir des faits. La féminisation de la gendarmerie (1983-2005), Vincennes, SHD, 2008.

30. Jean-Noël Luc (dir.), Figures de gendarmes, numéro spécial de Sociétés \& Représentations, 2003/16 ; Édouard Ebel, Yann Galera, Les gendarmes de la Belle Époque au miroir du Petit Journal, Vincennes, SHD, 2005 ; Histoire et dictionnaire de la gendarmerie, dir. Jean-Noël Luc et Frédéric Médard, Paris, Jacob-Duvernet, 2013, avec des chapitres sur les représentations des gendarmes dans la littérature, la BD, au cinéma et sur le petit écran.

31. Rennes, PUR, 2010.

32. Intitulé de l'un des axes du programme ANR SYSPOE, présenté plus loin.

33. Jean-Marc Berlière, Laurent Chabrun, Les Policiers français sous l'Occupation, Paris, Perrin, 2001 ; Benoît Majerus, Occupations et logiques policières. La police bruxelloise en 1914-1918 et 1940-1945, Bruxelles, Académie Royale de Belgique, 2008 ; Jonas Campion, Les Gendarmes belges, français et néerlandais à la sortie de la Seconde Guerre mondiale, op. cit. 
et notamment des fonctions policières, judiciaires et pénitentiaires des prévôtés ${ }^{34}$, longtemps totalement méconnues, ainsi que de ses contributions spécifiques aux opérations ${ }^{35}$, élargit le champ de l'histoire des armées et de la défense. La thèse publiée de Louis Panel, La Grande Guerre des gendarmes. "Forcer, au besoin, leur obéissance ${ }^{36}$ », analyse toutes les activités des territoriaux et des prévôtaux (contrôle des militaires en temps de paix, organisation de la mobilisation, police des combattants et de « l'arrière immédiat du champ de bataille », renseignement, protection des civils, pacification des territoires occupés, gestion de la sortie de guerre), ainsi que leurs représentations négatives et leurs relations par moments très difficiles avec les autres soldats.

\section{Donner un visage aux forces de l'ordre}

L'approche institutionnelle constitue le soubassement du chantier. Comment comprendre les comportements des agents, jusque dans leurs écarts à la norme, si l'on connaît mal les objectifs officiels, la réglementation, la gestion et les moyens, humains, financiers et matériels, des corps respectifs ? L'étude des rouages et du fonctionnement de la gendarmerie et des polices n'est pas démodée. Elle permet aussi d'observer, sous un angle original, la rivalité ou la collaboration des autorités centrales et périphériques autour de la tutelle de deux instruments de pouvoir emblématiques. Elle tire de l'ombre des organismes mal connus, à l'image des services de la police coloniale ou post-coloniale. Elle est indispensable pour comprendre l'expansion et la diversification de deux institutions qui multiplient, et de plus en plus à partir des années 1950, les unités spécialisées.

Les forces de l'ordre se définissent encore par les images qu'elles donnent d'elles et par celles que les populations leur renvoient ${ }^{37}$. L'histoire de ces représentations n'a pas pour vocation de produire un florilège de discours, d'écrits, d'images fixes ou animées et d'objets contradictoires, propres à nourrir les légendes noires ou roses de chaque corps. Pour le bonheur du chercheur, les personnages créés par un chansonnier, un dessinateur, un cinéaste ou par des communicants ne sont pas des copies totalement conformes des gendarmes et des policiers - d'ailleurs très divers - d'une époque donnée, même s'ils leur empruntent plusieurs de leurs traits. Ce sont des figures modelées, pour les besoins de l'art, de la parodie, de la polémique, de la publicité ou d'une politique de sécurité, et sélectionnées ou hiérarchisées en fonction des publics cibles. Traqueur des insoumis, policier politique, briseur de grève, planqué, brave pandore, serviteur de la loi, enquêteur, secouriste : autant d'archétypes dont l'étude exige de contextualiser les usages. À l'historien d'identifier les enjeux et les mutations d'une mise en scène qui se déploie dans le temps long et la conjoncture : ils constituent un indicateur original du rejet ou de l'acceptation de l'une

34. Sur l'action des prévôtés, ces unités de gendarmes détachées auprès des autres troupes : Olivier Buchbinder, Gendarmerie prévôtale et maintien de l'ordre (1914-1918), Maisons-Alfort, SHGN, 2004 ; Hélène de Champchesnel, La Gendarmerie au Levant pendant la Seconde Guerre mondiale, à paraître en 2014 (SHD) ; Aziz Saït, Les Prévôtés, de la « drôle de guerre » à «l'étrange défaite ». Personnels, missions, représentations, 1939-1940, doctorat, Paris-Sorbonne, dir., Jean-Noël Luc, 2012, et le doctorat en préparation, au même endroit, de Pierre-Yves Le Quellec, Les Prévôtés sur les théâtres d'opérations extérieures en Extrême-Orient, de la Guerre d'Indochine à la Guerre de Corée.

35. Gildas Lepetit, «La Manière la plus efficace de maintenir la tranquillité »? L'intervention de la gendarmerie impériale en Espagne (1819-1814), doctorat à paraître en 2014 (PUR-SHD).

36. Paris, Nouveau Monde éditions-DMPA, 2013.

37. Aux références citées supra, il faut ajouter Jean-Marc Berlière, « Images de policiers : deux siècles de fantasmes », Annuaire d'histoire administrative européenne, n 6, 1994, p. 125-148; Olivier Philippe, La Représentation de la police dans le cinéma français (1965-1992), Paris, L'Harmattan, 1999, et l'Histoire et dictionnaire de la police, op. cit. 
ou l'autre force, des politiques successives d'ordre public, des attentes sociales et de leur médiatisation $^{38}$.

Indispensable à la compréhension des stratégies officielles, des identités professionnelles et des réactions de la population, l'analyse des représentations des gendarmes et des policiers ne répond pas à l'une des questions clés du renouvellement historiographique : que font-ils vraiment ? On ne reviendra pas ici sur les démarches fécondes d'un Monjardet, relayées par celles d'un Napoli. À côté de cette première question, essentielle, l'histoire sociale, elle aussi renouvelée, en ajoute d'autres. Qui sont ces agents, militaires ou civils, de l'ordre public ? Comment sont-ils recrutés et formés ? Comment vivent-ils ? Quelles interactions construisent-ils avec les autres agents de la sécurité publique et avec leurs administrés ?

Contrairement à l'opinion de Michel Foucault, qui réduisait les représentants de l'État à des «machinistes dont le nom et le visage importent peu ${ }^{39}$ », il faut s'intéresser, et sur une grande échelle, aux hommes et aux femmes ${ }^{40}$ qui donnent corps aux institutions policières. Comment décoder les procès-verbaux si les conditions de l'apprentissage du " métier de gendarme » restent inconnues ? Comment interpréter les attitudes des agents de l'ordre à l'égard des différentes catégories de la population, et leurs propres définitions des seuils de l'admissible, si l'on ignore la distance ou la proximité - géographique, sociale, communautaire, linguistique, familiale, économique - entre ces représentants de l'État et leurs administrés ? Comment comprendre les tensions, les revendications, feutrées ou bruyantes, relayées ou entretenues par les organisations de retraités, les syndicats, les médias, ainsi que les crises, sans prendre en considération les effets générationnels, les voies d'accès aux corps, les systèmes de promotion, les carrières, les rémunérations, les situations familiales ? Le chantier est hélas peu développé, et notamment car il réclame l'exploitation statistique d'une énorme documentation, composée, en dehors des sources démographiques habituelles, des registres matricules, des dossiers individuels (pension, épuration, « victimes du devoir ») et des registres de punitions. C'est dire la valeur des trop rares travaux publiés, et qui s'appuient sur des bases de données d'ampleur variable ${ }^{41}$.

38. Pour un programme de recherche : Jean-Noël Luc, « Du bon usage de l'histoire des représentations des gendarmes », Sociétés \& Représentations, op. cit., p. 5-36.

39. Michel Foucault, «La poussière et le nuage », L'Impossible Prison : recherches sur le système pénitentiaire au XIX $X^{e}$ siècle, dir. Michèle Perrot, Paris, Le Seuil, 1980, p. 37.

40. À l'ouvrage déjà cité sur La Féminisation de la gendarmerie..., est venu heureusement s'ajouter celui de Geneviève Pruvost, De la « sergote » à la femme flic. Une autre histoire de l'institution policière (1935-2005), Paris, La Découverte, 2008.

41. Par exemple, et du XIX ${ }^{\mathrm{e}}$ au XX $\mathrm{XX}^{\mathrm{e}}$ siècle : Arnaud Houte, Le Métier de gendarme, op. cit. (300 dossiers de retraite et un échantillon des registres matricules de sept départements); Édouard Ebel, Police et société, op. cit. p. 223-383 ; Aurélien Ligneureux, Servir Napoléon. Policiers et gendarmes dans les départements annexés, 1796-1814, op. cit. (un portrait de groupe des 620 commissaires de police et des 7317 gendarmes concernés) ; Vincent Denis, «Les commissaires de police parisiens de la chute de la monarchie à la Restauration », et Jean-Paul Jourdan, «Le profil social des commissaires de police : l'exemple de l'Alsace et de l'Aquitaine, 1800-1870 », dans Le commissaire de police au XIX ${ }^{e}$ siècle, dir. Dominique Kalifa et Pierre Karila-Cohen, Paris, Publications de la Sorbonne, 2008, p. 27-67; Luc Keunings, Les Forces de l'ordre à Bruxelles au XIXe siècle. Données biographiques illustrées sur les officiers de la police, de la garde civique et de la gendarmerie (18301914), Bruxelles, Archives de la Ville de Bruxelles, 2007 (où ne figure pas une volumineuse base de données) ; William Serman, Les Origines des officiers français, 1848-1870, Paris, Publications de la Sorbonne, 1979 ; Terry W. Strieter, « The Faceless Police of the Second Empire : A Social Profile of the Gendarmes of Mid-NineteenthCentury France », French History, 8-2, 1994, p. 167-195 (à partir des dossiers de pension de 720 gendarmes) ; Christian Chevandier, Policiers dans la ville, op. cit. (qui conjugue l'histoire sociale et individuelle grâce à un corpus, inhabituel, de 620 dossiers de « victimes du devoir » à Paris au cours du premier XX ${ }^{\mathrm{e}}$ siècle) et le doctorat en préparation, à Paris-Sorbonne, de Charles Chester, Commissaires de police et officiers de gendarmerie du XX 
La rencontre des historien(ne)s avec des gendarmes et des policiers ayant réellement existé bouscule plusieurs idées. Diffusé dès la naissance, en 1907-1908, des brigades mobiles de police judiciaire, l'argument du recrutement parmi des spécialistes rompus à la poursuite des malfaiteurs les plus dangereux ne résiste pas à l'examen des fiches de carrière des inspecteurs et des commissaires de la nouvelle formation. Les différences entre gendarmes et policiers ne sont pas non plus aussi tranchées que le directeur de la Sûreté générale l'affirme, à la même époque, dans le but de favoriser la démilitarisation du recrutement policier. Entre « le gardien de la paix à la ville et le gendarme au village [...], la ressemblance est frappante et complète ", signale au contraire un publiciste, quelques années plus tôt $t^{42}$. L'étude des itinéraires éclaire ce phénomène, puisqu'une part notable des policiers municipaux est recrutée parmi les démissionnaires ou les retraités de la gendarmerie. À Paris, les candidatures de gardes républicains aux emplois réservés de la préfecture de police montrent même une hémorragie continue et croissante au bénéfice de cette institution durant la Troisième République. En amont de ces transferts, l'association quotidienne des militaires aux gardiens de la paix pour les patrouilles et les opérations de maintien de l'ordre favorise une certaine porosité des cultures professionnelles entre deux corps apparemment dissemblables. À la fin de la Belle Époque, les gardes républicains envient les améliorations, réelles ou supposées, des conditions de travail et de rémunérations des agents de police, tandis que le préfet Lépine essaie d'imprimer à ses hommes une martialité gendarmique et que des polices municipales organisent des unités montées sur le modèle de la Garde et copient son uniforme ${ }^{43}$.

\section{La force publique comme système ?}

« La police et la gendarmerie sont deux autorités qui concourent au même but ${ }^{44}$ », estime, en 1877 , un policier parisien. L'affirmation invite à aller y voir de plus près, et d'abord en élargissant le regard au-delà des clivages institutionnels. C'est ce que fait, depuis 2009, le séminaire de Paris-Sorbonne, d'abord organisé autour de l'histoire de la gendarmerie, puis ouvert successivement à celle de la Sûreté, de la Police nationale et des polices municipales, à l'histoire des pompiers et, bientôt, à celle des Douanes. La deuxième étape est moins facile, puisqu'il s'agit de dépasser, dans un séminaire, un colloque ou une publication, la simple juxtaposition d'études relatives à des acteurs différents. C'est ce que réussit, en 2002, Aurélien Lignereux dans l'ouvrage tiré de sa maîtrise, Gendarmes et policiers dans la France de Napoléon. Le duel Moncey-Fouché ${ }^{45}$, qui propose, au-delà de la "guerre des chefs », une « histoire relationnelle » complète. L'étude des rapports et des frictions, aux niveaux respectifs des bureaux parisiens et des hommes de terrain, montre comment l'instrumentalisation impériale d'une concurrence contrôlée produit la matrice des rivalités et des complémentarités consubstantielles à la dualité policière française.

Car pour donner tous ses fruits, l'approche transversale requiert de ne pas se focaliser sur les seules tensions, réelles ou fantasmées, et déjà présentes au sein de chaque corps. L'histoire des interactions entre les forces de l'ordre doit s'intéresser à toutes leurs relations,

siècle. Prosopographies comparées des commissaires parisiens et des officiers de la région parisienne à partir des dossiers de l'épuration et des dossiers personnels (un travail entrepris dans le sillage d'un master II qui a déjà permis de collecter entre 60 à 100 informations différentes pour 168 commissaires).

42. Ernest Leblanc « La gendarmerie, son histoire et son rôle. Les inconvénients du régime mixte », $L a$ Nouvelle Revue, mai-juin, 1880, p. 562.

43. Laurent López, La Guerre des polices n'a pas eu lieu, op. cit., à paraître.

44. M. F. Mironneau, Nouveau manuel de police judiciaire et administrative ou guide pratique à l'usage des officiers de police judiciaire et agents de l'autorité et de la force publique, Paris, Fayard, 1877, p. 243.

45. Maisons-Alfort, SHGN. 
donc aussi à leur coopération. Peu d'auteurs ont adopté cette démarche sur une grande échelle, du moins en histoire contemporaine ${ }^{46}$, avant la thèse pionnière, soutenue en 2012 et déjà citée, de Laurent López, qui, en explorant l'ensemble du territoire français, avec des détours par la Belgique, la Suisse ou le Luxembourg, analyse la coproduction de la force publique par les gendarmes et les policiers entre 1870 et 1914.

Depuis le $1^{\text {er }}$ janvier 2013, le programme ANR Histoire des systèmes policiers en Europe, XVIII ${ }^{e}$-XIX ${ }^{e}$ siècles (SYSPOE), auquel collaborent plusieurs chercheurs étrangers, permet d'étendre l'enquête, institutionnellement, géographiquement et chronologiquement. La notion de « système » recouvre ici «l'intégration selon un schéma hiérarchique entre différentes forces de police » et «l'articulation et les différentes interactions qui existent, dans un espace donné, entre les différents acteurs, civils ou militaires, publics ou privés, professionnels ou non, qui contribuent à la police comme activité. » L'étude est conduite sur des territoires variés, des situations d'interaction entre acteurs différents et des moments particuliers, qui «constituent, mettent en mouvement ou bousculent les systèmes policiers $^{47} \gg$.

En présentant l'ouvrage de Raymond B. Fosdick, European Police Systems, Ilsen About montre que la notion de «système policier» apparaît dès $1915^{48}$. Quels éléments accréditent la pertinence de ce terme pour désigner ce que constitue alors la force publique française ? Les conclusions de la thèse, déjà citée, sur les relations entre les gendarmes et les policiers français à la fin du XIX ${ }^{\mathrm{e}}$ siècle permettent d'apporter quelques pièces au dossier $^{49}$. Le rapprochement entre les premiers et les seconds sous le double aspect des représentations et des pratiques, ainsi que l'accélération d'une centralisation fonctionnelle et l'essor d'une culture judiciaire commune, figurent au premier rang de ces convergences. Car la préfecture de police et la Sûreté générale, ainsi que les gendarmeries, se sont indéniablement rapprochées depuis les années 1870. Leurs liens se sont consolidés tout en se multipliant ; leurs échanges se sont accrus; leur langage judiciaire connaît des essais d'homogénéisation ; leurs personnels respectifs se rencontrent plus fréquemment. Un embryon de système policier à l'échelle nationale semble bel et bien émerger de ce faisceau d'indices concordants.

Pourtant, dans les années 1870, les forces de l'ordre françaises étaient loin de constituer un tout organisé dont les éléments interagissent. Un tel système s'est d'abord formé sous la contrainte des faits divers et de la surveillance des anarchistes, qui ont stimulé, au sein de chaque force, la nécessité de se relier aux autres. En 1914, la transition d'un dispositif associant plusieurs institutions à une configuration systémique s'est opérée. Les décrets et autres règlements ont favorisé cette nécessaire collaboration, qui émerge également dans des micro-relations quotidiennes peu visibles. Dans et par ces liens, les individus ont élaboré des «systèmes partiels d'interaction ${ }^{50}$ », en créant les conditions de l'initiative

46. Édouard Ebel, Police et société [...] en Alsace, op. cit., p. 165-221 (un chapitre sur les rapports entre la police, la gendarmerie, les autres forces armées, la Justice, le préfet).

47. http://syspoe.hypotheses.org/le-programme-syspoe. Cinq axes orientent les recherches : « Polices plurielles », « systèmes policiers et espaces coloniaux », « culture militaire et systèmes policiers », " systèmes policiers, crises, révolutions et catastrophes » et « histoire circulatoire des systèmes policiers ». Le premier atelier international du consortium, intitulé «Qu'est-ce qu'un système policier ? », a permis, les 22 et 23 novembre 2013, de préciser les contours historiographiques et méthodologiques de cette notion.

48. Ilsen About, «Qu'est-ce qu'un système policier ? Le voyage de Raymond B. Fosdick à travers les polices d'Europe, 1913-1915 », dans Circulations policières (1750-1914), dir. Catherine Denys, Lille, PUS, 2012, p. 63-83.

49. Laurent López, La Guerre des polices n'a pas eu lieu..., op. cit.

50. Jacques Lagroye, Sociologie politique, Paris, Presses de la FNSP-Dalloz, 1993, p. 193sq. 
personnelle au sein d'un ensemble de contraintes, représentées en particulier par l'existence du corps concurrent, et désormais allié.

Un des obstacles à la désignation de la force publique est d'ordre terminologique. Comment appeler l'ensemble représenté par les forces de l'ordre : système, assemblage, appareil, institution, organisation, ensemble, machine, dispositif, instrument, pour citer les mots les plus fréquents ? Plus ou moins adapté, ou inadapté, selon les différents contextes, chacun de ces termes renvoie à des démarches méthodologiques dissemblables, dont la discussion sort de notre propos. On propose simplement ici une réflexion fondée sur l'approche dialectique des relations entre les gendarmes et les policiers français à la fin du XIX ${ }^{\mathrm{e}}$ siècle. Au-delà d'une simple description des circonstances ou des organisations juxtaposées, l'expression forte de « système policier », pourrait être alors entendue comme l'établissement institutionnalisé et durable de relations réciproques et diversifiées entre des agents relevant d'organismes ou de services différents. Produits, dans des proportions variables, de l'autonomie de ces agents, ces liens participent à l'accomplissement de missions aux finalités complémentaires, dont la somme forme l'ordre public, c'est-à-dire le fondement de l'État.

\section{Gendarmes et policiers sous le regard de l'histoire comparée et connectée}

« Nous avons pris cela des Français, et nous espérons, dans quelques années, avoir d'aussi bons résultats qu'eux ${ }^{51} »$. En quelques mots, prononcés en 1865 , ce responsable de la gendarmerie impériale mexicaine a tout dit : l'emprunt à l'étranger, à l'époque de l'éphémère empire de Maximilien, l'interrogation sur le résultat de la greffe et, pour nous, l'intérêt de l'histoire des transferts policiers.

Une étape est ici déterminante, du moins en ce qui concerne les initiatives françaises : le programme ANR Circulation des savoirs policiers européens, 1650-1850 (CIRSAP), coordonné par Catherine Denys et mis en œuvre entre 2006 et 2009. Étape ou plutôt acte fondateur, si l'on en juge d'après les problématiques comparatistes, le grand nombre de chercheurs associés, la collaboration avec les sociologues de la police et le legs d'une dynamique à partir de laquelle l'actuel programme ANR SYSPOE s'est élaboré. On appréciera mieux la contribution de ce chantier en rappelant, trop rapidement, ses grands objets d'étude : la circulation internationale, y compris dans les empires coloniaux, des écrits, des savoirs, des dispositifs et des usages policiers ; l'acceptation, l'adaptation ou le rejet des influences étrangères par les agents locaux ; la place méconnue des expériences exogènes dans des innovations habituellement rattachées à des idiosyncrasies municipales ou nationales européennes. L'enquête articule la macro et la micro-histoire, du réexamen de la construction et de l'audience des grands référents (parisien, londonien, caméraliste, métropolitain) jusqu'à la découverte des individus, passeurs ou opposants, qui assurent ou qui empêchent leur diffusion, leur imitation, leur adaptation ${ }^{52}$.

L'ouverture de CIRSAP sur l'histoire des polices ultramarines témoigne du dynamisme croissant de ce champ de recherche. L'analyse spécifique d'un maintien de l'ordre essentiel à la colonisation peut même apparaître comme l'un des moteurs récents du renouveau des

51. Adrien Kipeurt, « La gendarmerie au Mexique, 1861-1867 », Force publique, ${ }^{\circ}$ 7, septembre 2012 , p. $62 \mathrm{sq}$.

52. http://irhis.recherche.univ-lille3.fr/ANR-CIRSAP.html. À titre d'exemples : Catherine Denys, Brigitte Marin, Vincent Milliot (dir.), Réformer la police. Les mémoires policiers en Europe au XVIII siècle, Rennes, PUR, 2009 (le $2^{\mathrm{e}}$ volet de l'enquête après la publication, en 1996, de l'ouvrage déjà cité sur l'espace français) ; Catherine Denys (dir.), Circulations policières, op. cit. (avec neuf études de cas, étendues à New York et Buenos Aires) ; Vincent Denis et Catherine Denys (dir.), Polices d'Empire, XVIII ${ }^{e}$-XIX ${ }^{e}$ siècles, Rennes, PUR, 2012, qui offre la particularité de traiter des empires portugais, espagnols, hollandais et anglais. 
études coloniales. Si l'immensité des territoires, y compris archivistiques, à explorer est réelle, et le retard des travaux sur l'empire français, non moins évident, on préfère souligner l'activisme fructueux révélé par la succession des colloques ${ }^{53}$ et des publications. L'histoire de l'exercice de la police en terres coloniales, que les chercheurs anglophones appellent colonial policing, prolonge ou renouvelle l'étude des polices métropolitaines, de l'État et de la gestion des colonies dans une perspective impériale. Parmi les multiples pistes déjà ouvertes, on citera les panoplies disciplinaires, l'hétérogénéité des appareils militaropoliciers, l'implication des colonisés, la catégorisation et la hiérarchisation des populations, la contribution des innovations coloniales à la modernité policière, l'importation, en métropole, de pratiques de police en situation coloniale ${ }^{54}$.

Grâce aux panoramas historiographiques récents, en particulier ceux d'Emmanuel Blanchard ${ }^{55}$, on peut se contenter d'attirer ici l'attention sur deux chantiers significatifs. Créé, en 2009, le Groupe d'études sur les mondes policiers en Afrique (GEMPA) rassemble de jeunes chercheurs engagés, pour la plupart, dans l'histoire des forces et des dispositifs «d'ordre »- tel que les métropoles et les colons le conçoivent - aux colonies. Du premier ouvrage de ce groupe, essentiellement consacré à l'empire africain français, jusqu'à Madagascar, on retiendra l'élargissement de l'enquête aux forces armées classiques, aux corps indigènes ou à des organismes de renseignement et de coopération méconnus, ainsi que le souci, heureux, de produire une histoire « à hauteur d'hommes », qui tire de l'ombre des figures de policiers aux parcours singuliers ${ }^{56}$.

Le second exemple, le chantier ANR (2011-2014) Renouveau impérial des États ibériques : une globalisation originale, 1808-1930? (GLO-IBER), conduit vers d'autres territoires, où la problématique « Ordre et désordre impérial » a été jugée assez importante pour faire l'objet d'un axe de recherche autonome, sous la responsabilité de François Godicheau. La (re)construction d'un État impérial espagnol au XIX ${ }^{\mathrm{e}}$ siècle est étudiée à partir des circulations des catégories et des figures de l'ordre public et du désordre, ainsi que de l'implantation et des missions de la guardia civil et des autres forces, militaires ou policières, en Espagne, à Cuba, aux Philippines et à Puerto Rico ${ }^{57}$.

La visite des vestiges de l'empire espagnol permet de rencontrer l'un des acteurs de l'expansion impériale auquel les autres chantiers ne se sont pas vraiment intéressés : le gendarme. Et pourtant, dès sa création, le prototype français voyage ! D'abord, au service de la Révolution, puis pour veiller au salut d'un « Empire » qui n'en finit pas de s'étendre.

53. Récemment: Forces armées à l'époque de la décolonisation, 1940-1975 (Paris, 2012), Les Administrations coloniales et la "pacification » aux XIX et XX $X^{e}$ siècles (Paris, 2012), Policing Empires : Social Control, Political Transition, (Post-)Colonial Legacies (Bruxelles, 2013).

54. Jim House et Neil Mac Master, Paris 1961. Les Algériens, la terreur d'État et la mémoire, Taillandier, 2008 ; Emmanuel Blanchard, La Police parisienne et les Algériens (1944-1962), Paris, Nouveau Monde, 2011.

55. Emmanuel Blanchard, Quentin Deluermoz et Joël Glasman, « La professionnalisation policière en situation coloniale : détour conceptuel et explorations historiographiques », dans Métiers de police en situation coloniale, dir. Emmanuel Blanchard, numéro spécial de Crime, histoire \& sociétés, 2011/15, p. 33-53 ; Emmanuel Blanchard, «Conclusion. Les forces de l'ordre colonial entre conservatoires et laboratoires policiers, XVIII ${ }^{\mathrm{e}}$ et XIX ${ }^{\mathrm{e}}$ siècles », dans Polices d'Empire, op. cit., p. 171-188 ; Emmanuel Blanchard et Joël Glassman, « Le maintien de l'ordre dans l'empire français : une historiographie émergente », dans Maintenir l'ordre colonial. Afrique et Madagascar, XIX ${ }^{\mathrm{e}}-\mathrm{XX}^{\mathrm{e}}$ siècles, dir. Jean-Pierre Bat et Nicolas Courtin Rennes, PUR, 2013, p. 11-41. Pour un premier bilan de l'historiographie relative à l'empire britannique : Georgina Sinclair (ed.) Globalising British Policing. The History of Policing, 4, Abingdon, Ashgate, 2011.

56. Maintenir l'ordre colonial Afrique et Madagascar..., op. cit. Un deuxième ouvrage, consacré au renseignement colonial et post-colonial, doit paraître en 2014.

57. http://www.casadevelazquez.org/recherche-scientifique/programmes/les-communautes-dinteretpolitique/globiber/axes/news/les-comportements-face-a-letat-acceptations-et-refus-axe-3/. 
Deux ouvrages récents, et déjà signalés, traitent simultanément des gendarmes et des policiers, expatriés ou, pour une minorité d'entre eux, indigènes, chargés de participer à l'intégration des départements annexés ou à la gestion de certains États satellites ${ }^{58}$. En étudiant la situation des quarante-cinq nouveaux départements français, Aurélien Lignereux propose une histoire totale de l' « Empire des policiers », conduite sur plusieurs niveaux : transferts institutionnels et maillage des postes; profils, missions et vie quotidienne des personnels ; interactions avec les populations ; attitudes de ces dernières, véritable baromètre du rejet ou de l'acceptation de l'ordre français.

Les vingt-trois travaux déjà soutenus au sein du séminaire de la Sorbonne sur la présence et le rôle des gendarmes en Algérie, au Maroc, en Tunisie, en Martinique, en NouvelleCalédonie, au Levant et en Indochine constituent les premiers jalons d'un chantier sur leurs interventions en Outre-Mer, jusqu'à l'époque de la coopération. La plupart de ces travaux n'ont pas encore donné lieu à une publication; quatre doctorats sont en cours de préparation (sur les gendarmes au Cameroun, 1919-1960, au Sénégal, 1840-1870, au Liban, 1920-1939, et sur les prévôtaux pendant les guerres d'Indochine et de Corée), un doctorat soutenu va bientôt paraître (sur le Levant) ${ }^{59}$, trois autres, sur trois étapes de l'histoire de l'Algérie, ont été publiés.

«Sous le régime du sabre », de 1830 à 1870 , l'armée répugne au développement de la gendarmerie, que réclament les autorités civiles et judiciaires. Pour ces dernières, comme pour plusieurs responsables du corps, les gendarmes doivent montrer la supériorité de l'administration française sur la tutelle ottomane en protégeant les indigènes et en leur garantissant la justice ${ }^{60}$. De la fin des années 1930 à la répression des émeutes du Constantinois en mai 1945, la « gendarmerie du bled » et la garde républicaine mobile vivent à l'ombre de Vichy, réagissent de diverses manières au débarquement allié, participent au nouvel effort de guerre et multiplient les « mises en garde alarmantes », mais négligées, à propos du regain du nationalisme ${ }^{61}$. Pendant la Guerre d'Algérie, la gendarmerie, dont les magistrats demandent le renforcement, « apparaît comme un garde-fou face à une armée que les nécessités, réelles ou supposées, de l'action risquent d'entraîner trop loin ${ }^{62}$. » À ces trois doctorats, il faut ajouter une publication méconnue sur l'action, entre 1925 et 1934, des commandants du détachement de gendarmerie de la Nouvelle-Calédonie, par ailleurs nouveaux chefs du Service des affaires indigènes. À côté de certains compromis, ces militaires infléchissent les interventions de l'administration dans un sens favorable aux Mélanésiens, au grand mécontentement de plusieurs colons, déjà irrités par l'indépendance de ces gendarmes qu'ils accusent de « se moquer pas mal de la colonisation ${ }^{63}$. »

58. Police et gendarmerie dans l'Empire napoléonien, dir. Jacques-Olivier Boudon, op. cit. (avec une introduction historiographique de Jean-Noël Luc, « Les gendarmes de l'Empereur sous le regard des historiens ») et Aurélien Lignereux, Servir Napoléon. Policiers et gendarmes dans les départements annexés, 1796-1814, op. cit.

59. Helène de Champchesnel, La Gendarmerie au Levant pendant la Seconde Guerre mondiale, op. cit, à paraître en 2014.

60. Damien Lorcy, Sous le régime du sabre. La gendarmerie en Algérie, 1830-1870, Rennes, PUR, 2011 (doctorat, Bordeaux III).

61. Benoit Haberbusch La Gendarmerie en Algérie, 1939 à 1945, Maisons-Alfort, SHGN, 2004.

62. Emmanuel Jaulin, La Gendarmerie dans la Guerre d'Algérie, Paris, Lavauzelle, 2009, p. 401.

63. Jean-Marie Lambert, La Nouvelle politique indigène en Nouvelle-Calédonie. Le capitaine Meunier et ses gendarmes, 1918-1954, Paris, L'Harmattan, 1999, p. 68-89. Sans chercher à faire une anthologie, on signalera aussi la lutte du chef d'escadron Joseph France, commandant de la gendarmerie royale de la Martinique, entre 1843 et 1845, contre les sévices infligés aux esclaves et contre l'esclavage lui-même. La colère des colons aboutit au renvoi de l'officier en métropole, où il poursuit son combat abolitionniste. Emmanuel Frère, $L a$ 
Les travaux déjà réalisés et les recherches entreprises par des historiens étrangers ont permis à l'Université Paris-Sorbonne et à la SNHPG d'organiser, en juin 2013, un colloque sur Les gendarmeries dans le monde, de la Révolution à nos jours. Du Japon au Brésil en passant par le Siam, l'Iran, le Maghreb et l'Afrique noire, le destin des gendarmeries de vingt-six pays a éclairé sous un autre angle l'histoire, comparée et connectée, de la force publique et des systèmes policiers. Le chantier se poursuit en intégrant toutes les problématiques déjà citées : l'histoire n'a pas vocation à étalonner les gendarmeries du monde, y compris les héritières directes de l'arme française, à l'aune d'une matrice unique ! Mais l'appartenance des gendarmes à des institutions militaires bureaucratisées, et chargées de veiller simultanément à l'ordre public et à l'application des lois, oriente la recherche vers des questions particulières. On citera, à titre d'exemples, les échanges à l'intérieur d'un même espace impérial ou entre deux empires distincts ; les modalités de la représentation d'un pouvoir central, en dehors des grandes villes, par un seul agent polyvalent ; la dichotomie, les tensions ou les complémentarités entre forces militaires, polices et autres autorités civiles ; la voie étroite et contrastée de la civilisation des mœurs policières et de la gouvernance dans un contexte colonial. S'engager sur cette dernière piste, y compris au niveau d'une micro-histoire du quotidien, suppose de prendre ses distances avec deux regards sélectifs qui la disqualifient, a priori. La représentation de la gendarmerie comme un corps « naturellement » légaliste fait l'impasse sur ses compromissions au cours de son histoire métropolitaine et impériale. L'accent mis sur les seules formes très brutales, et incontestables, de la gestion coloniale laisse dans l'ombre l'action de militaires ou de civils, laïques ou religieux, engagés auprès des populations dans des tâches administratives, scolaires ou sanitaires.

\section{Pour une histoire de la force publique dans toutes ses missions et à toutes les échelles}

Les textes de ce dossier illustrent certaines des voies du renouvellement de l'histoire policière et ses contributions à l'histoire du renseignement, de la crise des années 1930, des relations entre les policiers et la culture de leurs administrés, de la gestion coloniale et de la coopération. Avant d'arrêter cette tournée historiographique, inhabituelle, en compagnie simultanée de gendarmes et de policiers, on jettera un coup d'œil sur quelques territoires encore vierges ou insuffisamment explorés.

Le cadre institutionnel montre son utilité en attirant l'attention sur des corps dont l'histoire reste à écrire, de la gendarmerie maritime (1791) ou de la police spéciale des chemins de fer (1855) à la gendarmerie des Forces Françaises en Allemagne (1945) et aux multiples unités ou services spécialisés de gendarmerie et de police, institués, à partir des années 1950, pour répondre aux besoins croissants et diversifiés de la sécurité et de la police judiciaire, de la police de la circulation ou de celle des milieux naturels. Il suffit de considérer la dénomination de ces nouveaux corps (CRS maîtres-nageurssauveteurs, pelotons de gendarmerie de haute montagne, unités de contrôle antipollution, gendarmerie des transports aériens) pour comprendre que leur étude monographique rencontrera forcément l'histoire générale de la société, et, ici, celle des loisirs, du tourisme, des transports et des risques, technologiques ou terroristes. La même voie d'entrée conduit vers les polices municipales, qui restent mal connues depuis le début du XIX ${ }^{\mathrm{e}}$ siècle, alors que de nouvelles monographies éclaireraient les spécificités locales de l'ordre public, les interactions entre ses agents et les enjeux politiques municipaux.

compagnie de gendarmerie de la Martinique (1820-1870), master, Paris-Sorbonne, dir. Jean-Noël Luc, 2001. p. 117-152. 
Même si les chantiers déjà cités ne peuvent ignorer les personnels, l'histoire sociale de certaines catégories de gendarmes et de policiers mérite des recherches autonomes. Les pistes neuves sont ici nombreuses, du recrutement des différentes écoles aux titulaires d'une fonction particulière, à une époque ou dans un lieu donné, en passant par les parcours individuels et les problématiques du genre. Parmi les policiers et les gendarmes réels que l'historien aimerait rencontrer, on citera les officiers et les commissaires de la Belle Epoque et de l'Entre-deux-guerres, les gendarmes départementaux et les gardiens de la paix des années 1950-1980 ou encore les états-majors des deux institutions, ainsi que les responsables des associations de retraités ou des syndicats au cours des années 1920-1960.

«La police pêche souvent par maladresse, par brutalité, par inintelligence, par manque de tact et de souplesse ! » Cet avis catégorique d'un juge de Bruxelles, en 1912, sur la conduite d'une enquête ${ }^{64}$, excitera légitimement la curiosité de l'historien pour la pratique policière elle-même. Sans négliger les travaux déjà réalisés ${ }^{65}$, on peut estimer que l'histoire de l'exercice des missions de la force publique depuis la fin du XVIII ${ }^{\mathrm{e}}$ siècle peut faire encore l'objet de nombreuses recherches, quand elle ne reste pas à écrire sur certains sujets. On connaît mal toutes les étapes et toutes les modalités de la diffusion de la police scientifique et technique dans les deux corps depuis la Belle Époque, ou les relations des policiers à leurs indicateurs en matière d'enquête. On n'est pas mieux renseigné sur l'ensemble des interactions entre les générations successives d'agents de l'ordre et de grévistes ou de manifestants, tout au long de la Troisième République, ou sur le fonctionnement, l'équipement et les pratiques, dans des contextes diversifiés, des unités de gendarmes mobiles et de CRS au cours des années 1950-1980. Le travail ne manque pas non plus du côté des missions militaires de la gendarmerie sur les théâtres européens, coloniaux ou des OPEX. Et l'extension de l'enquête aux interventions des gendarmes et des policiers territoriaux ou spécialisés dans la police de la voirie et de l'hygiène publique ou à l'occasion d'accidents, d'épidémies, d'épizooties et de catastrophes naturelles conduit l'histoire policière, par des voies encore inexplorées, vers les chantiers prometteurs de l'histoire de l'environnement et des risques ${ }^{66}$.

Ces pistes tiennent compte de la segmentation et de la territorialisation de la gendarmerie et de la police, ainsi que d'une grande partie de leurs archives, dont tout chercheur est obligé de s'accommoder. Au moins ces contraintes rappellent-elles qu'à l'heure de l'européanisation et de la mondialisation, fécondes, de l'histoire des polices, l'approche nationale, provinciale et même locale constitue, pour certains sujets, des points de départ, des voies de passage ou des terrains d'étude autonomes obligés. Combien de monographies ont été réalisées sur des brigades départementales ou sur des commissariats, pendant une période significative, entre le début du XIX ${ }^{\mathrm{e}}$ siècle et les années 1970 ? Une seule à notre connaissance - sur la brigade de Bergerac dans l'entre-deux-guerres - du moins parmi les

64. Ryckère (de), Raymond, La Police scientifique, Bruxelles, Ferdinand Larcier, 1912, p. 5.

65. À titre d'exemples et pour la seule histoire récente de la police judiciaire ou du maintien de l'ordre : Clive Emsley, Crime, police and Penal Policy : European experiences 1750-1940, Oxford, OUP, 2007 ; L'Enquête judiciaire en Europe au XIX ${ }^{\mathrm{e}}$ siècle, dir. Jean-Claude Farcy, Dominique Kalifa, Jean-Noël Luc, Grâne, Créaphis, 2007 ; Pierre Piazza (dir.), Aux origines de la police scientifique. Alphonse Bertillon, précurseur de la science du crime, Paris, Karthala, 2011 ; Benoît Haberbusch, Les Gendarmes face au crime dans l'Entre-deux-Guerres, La Crèche, Geste éditions, 2012 ; Laurent Lopez, La Guerre des polices..., op. cit. ; Thierry Forest, La Gendarmerie mobile à l'épreuve de mai 1968, op. cit., Simon Fieschi, Les Gendarmes en Corse, 1927-1934. De la création d'une compagnie autonome aux derniers bandits d'honneur, Vincennes, SHD, 2012.

66. Fabien Locher, Grégory Quenet, « Histoire environnementale », numéro spécial de la Revue d'histoire moderne et contemporaine, 56/4, 2009, et Pour une histoire du risque. Québec, France, Belgique, dir. David Niget et Martin Petitclerc, Rennes, PUR, 2012. 
cent trente-trois travaux du chantier de la Sorbonne consacrés à l'histoire de la gendarmerie. Les cellules de base de l'épithélium gendarmique et policier mériteraient pourtant de retenir l'attention, jusque dans leurs interactions, des historien(ne)s qui se veulent attentifs aux pratiques des institutions et de leurs agents. Sur cet autre front de la recherche, on découvre le destin des acteurs de base de la force publique, leurs activités réelles, masquées ailleurs par les interventions spectaculaires, les interrelations quotidiennes au sein du monde des polices et de la société locale, enfin « le procès de construction de l'État », puis son fonctionnement, « au ras du sol ${ }^{67} »$.

CEnTRE D' Histoire DU XIX E SIÈCle (PARIS I - PARIS-SORbONNE) ET CENTRE D’ÉTUDES SOCIOLOGIQUES SUR LE DROIT ET LES INSTITUTIONS PÉNALES

(CESDIP)

67. Jacques Revel, Jeux d'échelle. La micro-analyse à l'expérience, Paris, Gallimard-Seuil, 1996, p. 29. 